\title{
On the impact of phonon spectrum shifts of the hydrogen binding in $\mathrm{ZrH}$
}

\author{
M. Badea ${ }^{\mathrm{a}}$, R. Dagan and C.H.M. Broeders \\ Institute for Reactor Safety, Forschungszentrum Karlsruhe, Postfach 3640, 76021 Karlsruhe, Germany
}

\begin{abstract}
In the framework of the ECATS (Experiments Coupling Accelerator Target Sub-critical blanket) project within the EC FP6 IP-EUROTRANS a coupling of external sources with a TRIGA core had been considered. In order to simulate dynamical transients in ADS (Accelerator Driven Systems) the reactivity feedback coefficients should be accurately determined. Two reported independent experiments at RC-1 Casaccia and at Slovenian MARK II TRIGA reactor Ljubjana have shown an "anomaly" reactivity feedback coefficient at temperatures between $100-150{ }^{\circ} \mathrm{C}$. This increased negative reactivity feedback in the mentioned temperature domain is not predicted by the General Atomic (manufacturer of TRIGA Core) reactivity curve. In this study the influence of the phonon spectrum on the temperature dependant reactivity feedback coefficient is being analyzed in view of the experimental results and existing studies of Malik et al., Slaggie and others. In particular the scattering kernels of hydrogen bound in zirconium, and in general phonon spectrum shapes and changes are studied.
\end{abstract}

\section{Introduction}

The standard reactivity feedback curve provided by General Atomics (GA) [1] for TRIGA fuel appears to be inadequate in comparison with new experimental data, in particular in the range of $100-140{ }^{\circ} \mathrm{C}$. The reactivity measurements which were performed in RC-1 TRIGA Casaccia and Slovenian TRIGA Mark II core Ljubljana exhibited a significant increase of the negative reactivity feedback in this range. Moreover the two experiments were conducted with different fuels. The one in RC-1 with considerably highly burned up fuel whilst the Slovenian TRIGA experiment was done with fresh fuel.

Previous work by Mattes et al. [2], Badea et al. [3], have shown the sensitivity of different numerical parameters introduced in the NJOY [4] code which had mainly influence on the criticality values but could not explain the clear enhancement of the negative reactivity coefficient in the relevant temperature range.

In the current study the new energy grid of NJOY module THERMR is tested again for hydrogen bound in zirconium and also the sensitivity of the different phonon spectrum shapes having several/single shifted peaks, and depending on the hydrogen concentration.

The phonon states in metal hydrides appear to be affected by the hydrogen concentration [5]. Moreover the fine structure of the hydrogen admixture is associated with the optical peak [6]. The corollary of the above observation means that in the case of TRIGA fuel the phonon spectrum used could be not adequate. The hydrogen's stoichiometry is different from the one upon which the scattering data were generated and the structure of the fuel matrix might be to some extent deformed by the existence of the fuel.

Thereafter it was decided to evaluate the influence of changes in the intermolecular structure and forces in form of the deviation in the phonon distribution function. In particular, the effect of shifting the optical part of the phonon spectrum on the temperature dependent reactivity feedback is introduced.

\footnotetext{
${ }^{a}$ Presenting author, email: badea@irs.fzk.de
}

\section{Experimental results}

The TRIGA fuel elements for these reactivity measurements are cylinders of ternary alloy uranium-zirconium-hydride with $\mathrm{H}$-to- $\mathrm{Zr}$ atom ratio 1.7 and the total $\mathrm{U}$ of $8.5 \%$ of the mixture by weight for the Casaccia reactor and with $\mathrm{H}-$ to- $\mathrm{Zr}$ atom ratio 1.65 and the total $U$ of $12 \%$ for the Slovenian reactor and the same enrichment ( $20 \%$ enriched $\left.{ }^{235} \mathrm{U}\right)$. The fuel cladding consists of stainless steel AISI 304 of $0.05 \mathrm{~cm}$ thickness and $7.8 \mathrm{~g} / \mathrm{cm}^{3}$ density. There are two graphite cylinders of $38.11 \mathrm{~cm}$ length at the top and the bottom of the fuel rod.

The reactivity coefficient measurements performed within the TRADE project in the Casaccia RC-1 core [7] showed similar results to the experimental temperature dependent values measured at the Slovenian TRIGA core [8] although the fuel composition was different. The reactivity measurements in RC-1 were carried out with the existing core configuration, i.e., with burned-up fuel. The measurements at the TRIGA Mark II at Slovenia were performed with completely fresh uniform core. In both experiments the temperature was measured only in one or two locations within the core, from which an average temperature was evaluated. In figure 1 the reactivity feedback per degree is plotted. The curves are subjected to uncertainties of about 3 degrees in temperature and about $\pm 5 \mathrm{pcm}$ in the reactivity value [8]. All curves differ considerably from the GA curve between $110-150{ }^{\circ} \mathrm{C}$. The curves of the Slovenian core refer to the estimation of the total core averaged temperature based on measurements at point A located in the centre of the core (denoted in the figure with $T_{a}$ ) and based on two point measurements at the above centre point $\left(\mathrm{T}_{\mathrm{a}}\right)$ and in the first ring of the core $\left(\mathrm{T}_{\mathrm{b}}\right)$.

As it is shown in figure 1 the differences between the curves emphasizes the necessity of an improved multi-point temperature measurement to evaluate more accurately the influence of the spatial flux distribution and the fuel content (fresh or burned up fuel) on the reactivity feedback. Nevertheless, as can be seen in figure 1 all the measurements indicate a phenomenon which is not shown in the GA recommendations, namely the increase of the absolute value of the reactivity coefficient above $100{ }^{\circ} \mathrm{C}$. This emphasizes the necessity of 


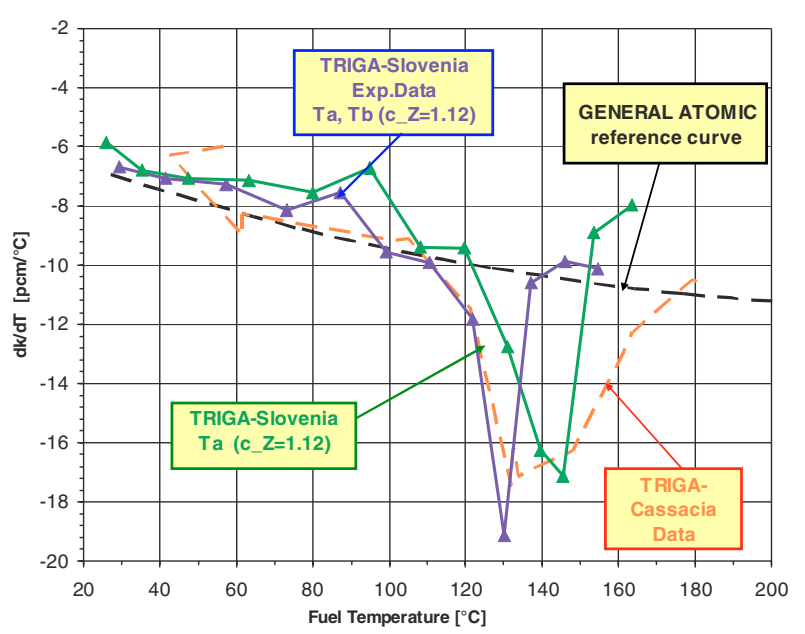

Fig. 1. Experimental temperature dependent reactivity feedbacks of the RC-1 and Slovenian TRIGA cores.

a comprehensive survey of the numerical as well as the fundamental physical treatments of the bound hydrogen in zirconium scattering kernel and their possible impact on the criticality and temperature reactivity feedbacks of TRIGA cores.

\section{The influence of the numerical treatment}

In previous studies [3] several numerical aspects with respect to the preparation of probability $\mathrm{S}(\alpha, \beta)$ tables for the secondary energy distribution concerning hydrogen bound in zirconium were analysed. It was shown that the tables based on the standard 59 energy grid structure resulted in erroneous criticality values for TRIGA fuel. The increase of the number of points in the energy grid (118) [4] based on the work of Mattes et al. [2] differed by about $300 \mathrm{pcm}$ in comparison with the standard grid as can be seen in figure 1. All the calculations in figure 2 and the following figures were performed with beta version of the MCNPX code with standard deviation of $7 \times$ $10^{-5}$. A new grid with 199 energy points was introduced in the current study taking into account all points from former studies dedicated to hydrogen bound in zirconium. These points were included in the NJOY updated grid definition [4]. The results deviate from the improved 118 points scheme by nearly $100 \mathrm{pcm}$ (fig. 2). A very fine, equal lethargy interval, energy grid with 2045 points, gave similar criticality values compared to the 199 points energy grid (fig. 2). This emphasizes the need to search further for an optimized grid for chemical binding of all isotopes of interest. The current general grid seems to be inadequate for handling all bounded light isotopes.

However, all the results presented so far can not reproduce the reactivity coefficients shown in figure 1 .

\section{The impact of a shifted phonon spectrum on the reactivity curve}

The phonon spectrum of hydrogen bound in zirconium suggested by Slaggie [6] and commonly used by NJOY [10] is

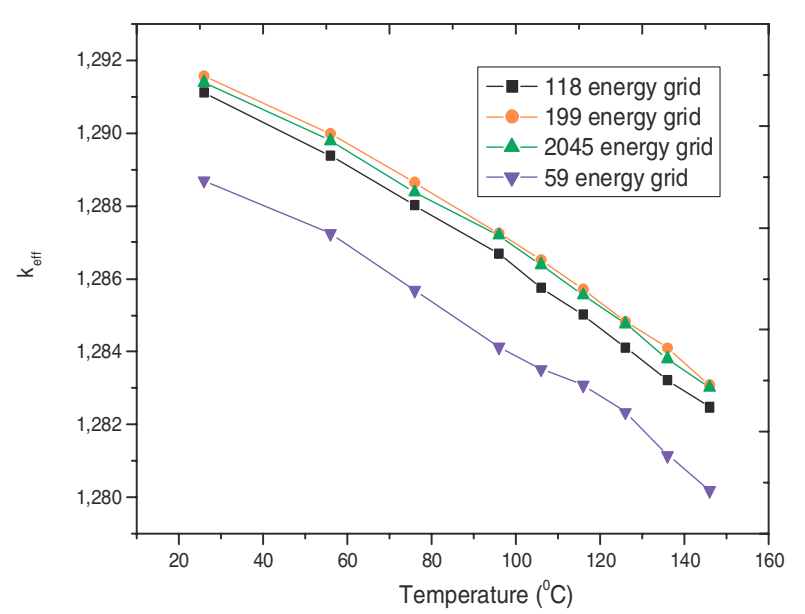

Fig. 2. Temperature dependent criticality values as a function of the energy grid points number.

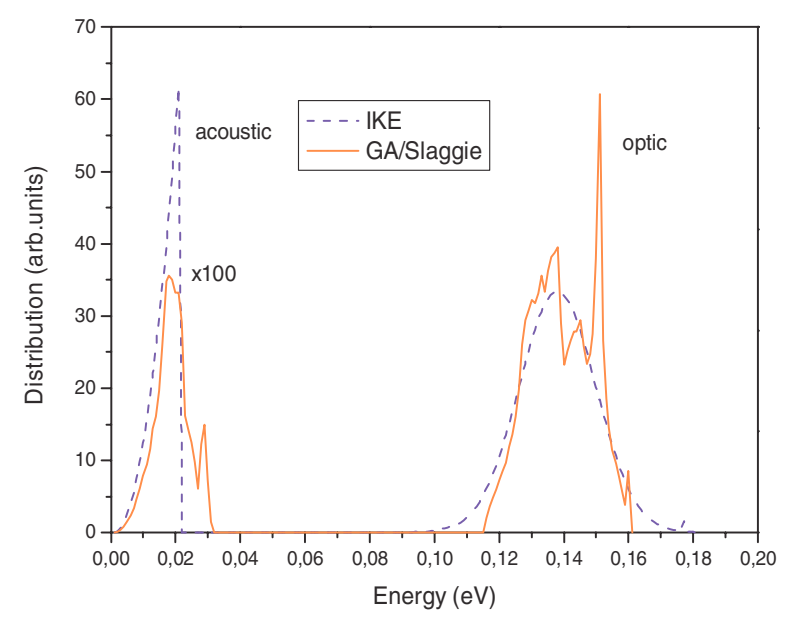

Fig. 3. Frequency distribution of $\mathrm{H}$ in zirconium (acoustic and optical part).

given in figure 3 . This spectrum is based on a central force model of $\mathrm{ZrH}_{2}$. Using it one gets in the low temperature region $\left(30-100{ }^{\circ} \mathrm{C}\right.$ ) good agreement in comparison to the reactivity measurements plotted in figure 1 . However, in the higher region $\left(100-140{ }^{\circ} \mathrm{C}\right)$ the shape of the measured reactivity coefficient curve is still not explained.

The work of Malik et al. [5] deals with the phonon spectrum of hydrogen bound in zirconium with an atomic ratio of $1.58\left(\mathrm{ZrH}_{1.58}\right)$. Malik describes in his work a phonon spectrum which is represented by three Gaussians with peaks at $0.132,0.137$ and $0.151 \mathrm{eV}$. He discusses the impact of the stoichiometry on the binding structure and the $\mathrm{H}-\mathrm{H}$ interaction which could give a better insight also for the $\mathrm{ZrH}_{2}$ model. Mattes referred to new experimental data from Evans [9] for $\mathrm{ZrH}_{2}$. In figure 4 Evans's experimental optical mode curve and its equivalent spectrum suggested by Mattes with one Gaussian peak at $0.137 \mathrm{eV}$, are presented. This proposed spectrum agrees better with relevant scattering measurements in comparison with Slaggie's model.

Different phonon spectrum shapes having several/single shifted peaks, and depending on the hydrogen stoichiometry, 


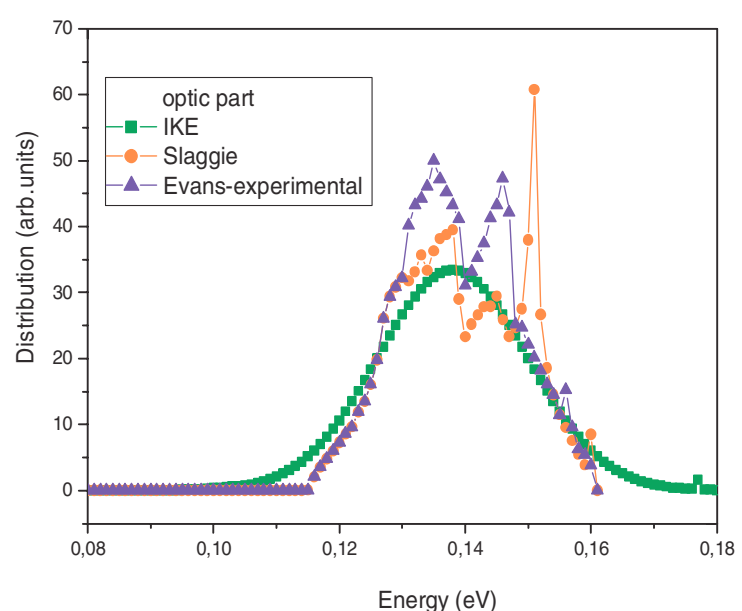

Fig. 4. Frequency distribution theoretical and experimental (optical part).

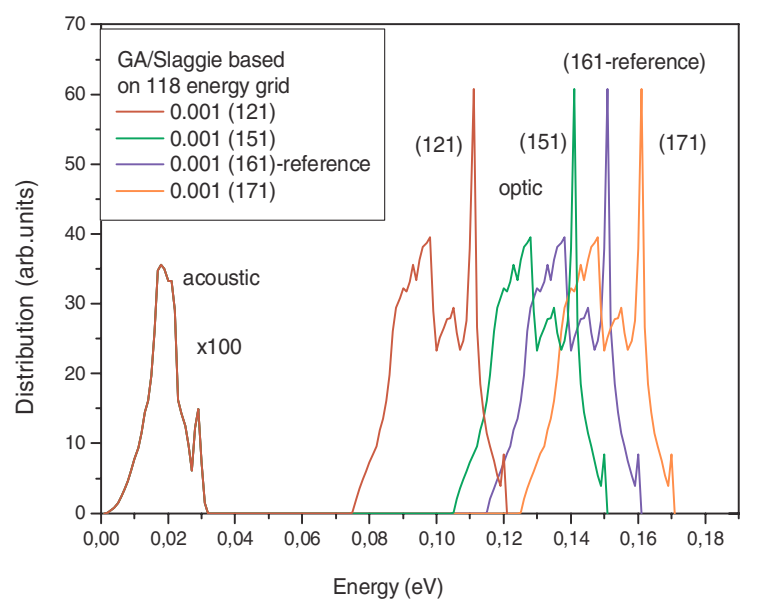

Fig. 5. Shifted phonon spectrums around the original one (161 energy intervals).

leads obviously to different scattering phenomena, with possible impact on the reactivity coefficient.

In the current experiments of RC-1 and Slovenian cores the atomic ratio is 1.7 and 1.65 respectively for which the spectrum is only approximated from other stoichiometry as discussed before. The TRIGA experiments were performed for hydrogen bound in TRIGA fuel and not only in zirconium (for which the data is generated). As a corollary, the actual phonon spectrum in TRIGA cores might be slightly deformed or shifted.

In this study potential deviation of the phonon spectrum from the reference curve [6] are analysed in view of their impact on the criticality and the temperature dependent reactivity feedbacks.

In figure 5 the various shifted optical spectra are plotted. The 161 energy points represent the original $0.137 \mathrm{eV}$ phonon peak. The "121", "151" titles stand for a shifted peak by 0.04 and $0.01 \mathrm{eV}$ respectively downwards where as the "171" introduce a phonon shifted $0.01 \mathrm{eV}$ upwards.

Decreasing the phonon spectrum energy tends in principle to enlarge the criticality which is in accordance with the results with the free gas model where the phonon peak location

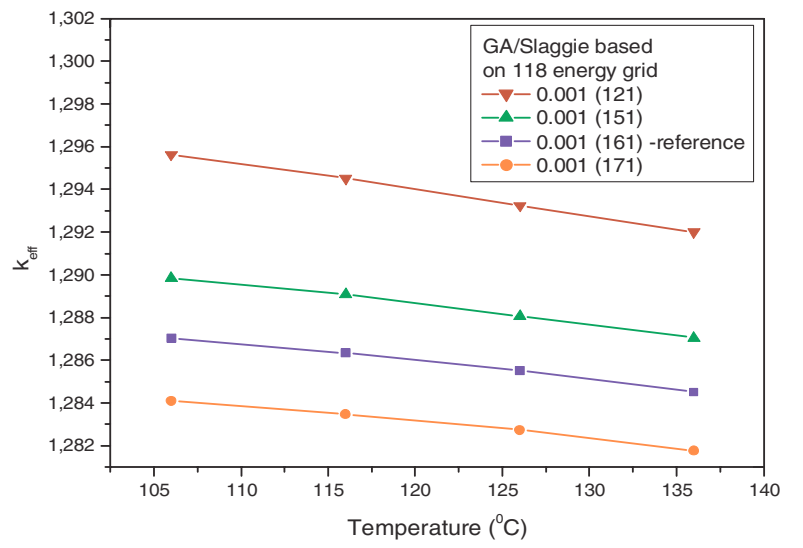

Fig. 6. Criticality calculations with different phonon spectrums for TRIGA fuel assembly.

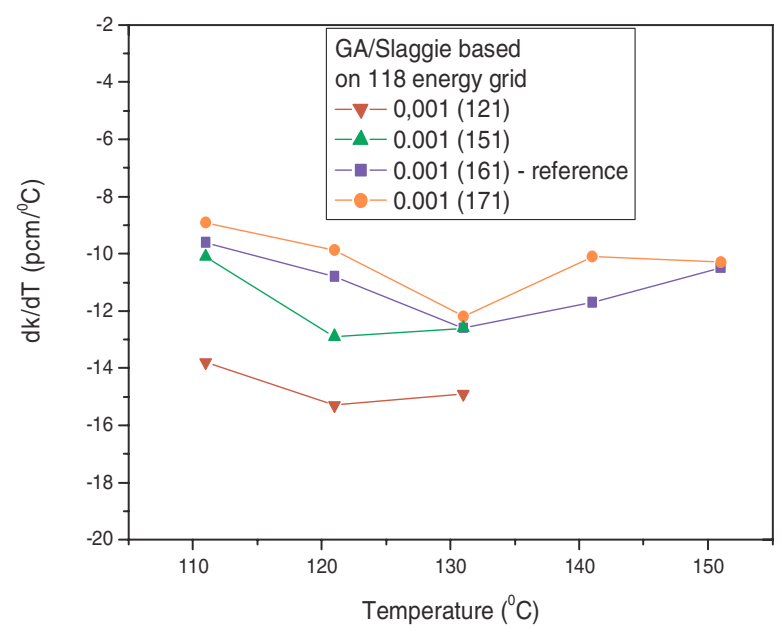

Fig. 7. The impact of different phonon spectrum (optical part) in the reactivity feedback.

approaches 0 . In order to replace experimental results one may assume that around $100^{\circ} \mathrm{C}$, due to currently unknown phenomena, the spectrum is shifted to higher energies by about $0.01 \mathrm{eV}$ and then the new spectrum peak vibrates in the about $0.147 \mathrm{eV}$. If this shift is taking place within the temperature range $\left(100-150^{\circ} \mathrm{C}\right)$, the corresponding criticality values shown in figure 6 , can explain the measurements of the reactivity coefficients depicted in figure 1 . The temperature dependent criticality derivation, which can be deduced from figure 6 , fits the reference curve (161) of the measured reactivity shown in figure 1 up to about $115^{\circ} \mathrm{C}$. A postulated shift in the phonon spectrum will cause a significant decrease in $\mathrm{k}_{\text {eff }}$, namely a transfer from a point on the reference curve (161) to another temperature dependent point on the (171) curve, figure 6. This "jump" is in accordance with the pronounced negative slope of the reactivity coefficient in the temperature domain $115-130^{\circ} \mathrm{C}$ in figure 1 .

The curves in figure 7 demonstrate that the reactivity coefficients for constant phonon spectrum have again only weak dependency on temperature as in the domain below $110^{\circ} \mathrm{C}$. Consequently, the reactivity coefficient will retain its previous level as is depicted in figure 1 . The feasibility of such a postulated shift is realistic and in accordance with 
the conclusions of Malik [5] and the work of Slaggie [6] concerning the contribution of $\mathrm{H}-\mathrm{H}$ forces to the vibration mode. Nevertheless, it is evident that more experiments and verifications are mandatory in respect to feedback experiments within the temperature range $100-150{ }^{\circ} \mathrm{C}$. Such investigations could contribute to an improved model of the varying stoichiometry of the bound Hydrogen in zirconium embedded in a fuel matrix.

\section{Conclusions}

This work extends a previous study [3] concerning the numerical and physical treatment of scattering kernel models for criticality calculations of TRIGA fuel. The improved energy grid scheme in the NJOY update 112 for module THERMR is not completely adequate and about 80 more points seem to be needed for hydrogen bound in zirconium.

The postulated deviation of the phonon spectrum in the temperature range $100-140^{\circ} \mathrm{C}$ explains the specific behaviour of the temperature dependant reactivity coefficient in the TRIGA subassembly. Theoretical results are presented, based on shifting the phonon spectrum. The sensitivity of reactivity feedbacks to changes in the spectrum is well pronounced in those calculations. Nevertheless, an experimental based physical confirmation is needed including also detailed measurements of hydrogen bound in a fuel matrix with zirconium as all current data deal practically with hydrogen bound in zirconium ignoring the fact the hydrogen is mixed within the fuel.

The stoichiometry and the intermolecular forces have mainly influence on the criticality. They should be merged together with the spectrum effect mentioned above. The modified scattering kernel for $\mathrm{ZrH}_{x}$, based on the idea of temperature dependent shift of the phonon spectrum agrees better with the experimental data shown in figure 1. This improvement is of high interest if TRIGA reactors will be applied for fundamental neutron physics experiments.

\section{References}

1. General Atomics, TECNICAL FOUNDATIONS OF TRIGA, GA-471, 1958.

2. M. Mattes, J. Keinert, Thermal Neutron Scattering Data for the Moderator Materials $\mathrm{H}_{2} \mathrm{O}, \mathrm{D}_{2} \mathrm{O}$ and $\mathrm{ZrH}_{x}$ in ENDF-6 Format and ACE Library for MCNP(X) Codes, INDC (NDS)-0470, April 2005.

3. M. Badea, R. Dagan, C.H.M. Broeders, Improved $S(\alpha, \beta)$ for TRIGA Criticality and Reactivity Feedbcak Calculations, Mathematics and Computation, Supercomputing, reactor Physics and Nuclear and Biological Applications, Avignon, France, 2005.

4. R. MacFarlane, The NJOY Nuclear Data Processing System, Version 99 (up112), 2005.

5. S.S. Malik et al., Optical-phonon structure and precision neutron total cross section measurements of zirconium hydride, J. Phys. F: Met. Phys. 14, 73 (1984).

6. E.L. Slaggie, Central Force Lattice Dynamical Model for Zirconium Hydride, General Atomic Report GA-8132, July 1967.

7. The Working Group on TRADE (TRIGA Accelerator Driven Experiment), TRADE Final Feasibility Report, March 2002.

8. I. Mele, M. Ravnik, A. Trkov, TRIGA MARK II Benchmark Experiment, Part I: Steady State Operation, Nuclear Technologies, Vol. 105, p. 37, Jan. 1994. Data evaluated by M. Schikorr FZK (private communication).

9. A.C. Evans et al., Neutron-scattering study of the impulse approximation in $\mathrm{ZrH}_{2}$, Phys. Rev. B 53, 3023 (1996).

10. R. MacFarlane, New Thermal Neutron Scattering Files for ENDF/B-VI Release 2, 1994. 